\title{
Left ventricular diastolic dysfunction in normotensive postmenopausal women with type 2 diabetes mellitus
}

\author{
Maria Maiello ${ }^{1}$, Annapaola $Z_{i t o}{ }^{2}$, Annagrazia Cecere ${ }^{2}$, \\ Marco Matteo Ciccone ${ }^{2}$, Pasquale Palmiero ${ }^{1}$ \\ ${ }^{1}$ ASL Brindisi, Cardiology Equipe, District of Brindisi, Italy \\ ${ }^{2}$ Cardiovascular Diseases Section, Department of Emergency and \\ Organ Transplantation (Deto), University of Bari, Italy
}

\begin{abstract}
Background: The prevalence of heart failure among diabetic patients is high, also in those with normal blood pressure and without coronary artery disease, even when electrocardiogram (ECG) is normal. The goal of our study was to assess the prevalence of left ventricular diastolic dysfunction (LVDD) among diabetic women $(D W)$ and its correlation with glycosylated hemoglobin (HbA1c) levels, obesity status, and ECG parameters.

Methods: A group of 456 consecutive normotensive postmenopausal women affected by type 2 diabetes, diagnosed over 5 years, were enrolled. One hundred normotensive non-diabetic postmenopausal women were included as a control group (CG). Rest ECG and trans-thoracic echocardiogram and Doppler were performed.

Results: LVDD was present in 103 (23.3\%) out of $456 \mathrm{DW}$, and 8 out of 100 women in CG (8\%), $p<0.001$. There was no difference in mean age between the two groups: $56 \pm 13$ and $55 \pm 3$, respectively $(p=0.3)$. There were $191(41.9 \%) D W$ with body mass index $(B M I)>30 \mathrm{~kg} / \mathrm{m}^{2}$. Among those, there were $56(12.3 \%)$ with significant prevalence of LVDD, while there were 49 (10.7\%) with BMI $<30 \mathrm{~kg} / \mathrm{m}^{2}, p<0.005 . \mathrm{DW}$ with HbA1c $>7.5 \%$ comprised a group of $243(53.3 \%)$ patients. Among those, there were 45 (9.9\%) with higher prevalence of LVDD, and 15 (3.3\%) with HbAlc < 7.5\%, $p<0.01$. Out of a group of 147 (32.2\%) DW with abnormal ECG, 21 had LVDD (4.6\%), $p=0,1$, and 84 (18.8\%) had LVDD with normal ECG.

Conclusions: Our data prove a high prevalence of LVDD in asymptomatic diabetic postmenopausal women. This finding is closely related with HbA1c levels and obesity status, not with abnormal ECG, which is a unique cardiologic test recommended by current guidelines in all diabetic patients. We conclude that early detection of high level of HbA1c and obesity $\left(30 \mathrm{~kg} / \mathrm{m}^{2}\right)$ may identify women with major risk to develop LVDD. Furthermore, a simple ECG, when normal, is not enough to assess a normal LV diastolic function. (Cardiol J 2017; 24, 1: 51-56)
\end{abstract}

Key words: left ventricular, diastolic function, type 2 diabetes, postmenopausal women

\section{Introduction}

Diabetes mellitus (DM) is the most common metabolic disease, especially in Western countries and its prevalence will double in the next 25 years [1].
Cardiovascular diseases are the leading cause of death among diabetic patients because, as widely demonstrated, chronic hyperglycemia results in morphological and functional changes on vascular wall, which leads to development of atherosclerotic plaque [2].

Address for correspondence: Pasquale Palmiero, MD, 72100, Brindisi, Italy, via Francia 47, fax +39 0831536556 , e-mail: pasqualepalmiero@yahoo.it

Received: 04.04.2016 Accepted: 14.08.2016 
It is mandatory to detect "diabetic cardiomyopathy" at an early stage, to improve cardiovascular risk stratification on diabetic patients. Left ventricular (LV) diastolic dysfunction (LVDD), without impairment of systolic function, i.e. with preserved LV ejection fraction (LVEF), is the first stage of "diabetic cardiomyopathy" [3]. The gold standard for LVDD assessment is echocardiogram with pulse wave (PW) tissue Doppler that allows to perform a non-invasive, rapid, free of radiation, reproducible assessment of $\mathrm{LV}$ filling and pressure $[4,5]$. Early LVDD of lower degree, as impaired relaxation pattern, can be considered a preclinical stage of disease, the heart reduces LV compliance and elevates filling pressure, increases left atrial contribution to LV filling, preserving LVEF [6]. It is quite clear that an impaired ventricular relaxation can determine, with time, a diastolic heart failure with a preserved ejection fraction (heart failure with normal ejection fraction, $\mathrm{HFpEF}$ ), with a mortality rate similar to systolic heart failure [7].

Pathogenesis of impaired LV relaxation in diabetic patients is not fully understood, although microvascular disease, autonomic dysfunction, other concomitant metabolic disorders, long duration of DM and poor blood glucose control are universally recognized as concurrent factors [8]. Microcirculation plays an important role in impairment of $\mathrm{LV}$ compliance, similarly to prolonged hyperglycemia causing fibrosis and autonomic dysfunction, with an additive and adverse effect of DM on cardiovascular system. Mishra et al. [9] found that DM represents the most common risk factor involved in the development of LVDD [9].

A significant amount of scientific evidence suggests a higher prevalence of LVDD in postmenopausal women [7-10] - a drop in estrogen causes a lack in physiological protection of endothelium with growth of atherosclerotic plaques and activation of the renin-angiotensin-aldosterone system (RAAS), with increase of angiotensin II and production of reactive oxygen species (ROS), with impaired ventricular relaxation [11].

It may be observed that in such patients, occur unfavorable changes in arteries structure and function [12]; aortic stiffness is an early marker of cardiovascular disease and atherosclerosis, [13] whose increase is often associated to abnormal LV filling [14]. Therefore, aortic stiffness increases with age and its association with mortality is twice higher in women than in men [15]. Postmenopausal women present a close correlation between aortic stiffness and hypertension, DM and hypercholesterolemia, indepen- dently of age when the menopause started [16]. According to these, aortic stiffness plays a prognostic role in identifying risk of developing adverse cardiac events along time.

The goal of our study is to evaluate the prevalence of LVDD in asymptomatic and normotensive postmenopausal women with DM, over 5 years. Our secondary endpoint was the correlation between LVDD and long-term glycemic control, assessed by glycosylated hemoglobin (HbA1c), obesity (body mass index [BMI] $>30 \mathrm{~kg} / \mathrm{m}^{2}$ ), being LVDD often associated with metabolic disorders.

\section{Methods}

\section{Patients}

A group of 456 consecutive normotensive postmenopausal women affected by type 2 DM over 5 years were enrolled. Menopause was assessed if occurred 12 months after last menstrual period, DM duration average time was $7 \pm 3$ years, all of them were on oral antidiabetic therapy, 45 of them were also on insulin treatment. Subjects with systolic dysfunction (LVEF $<50 \%$ ), mild-severe valvular diseases and atrial fibrillation were excluded. One hundred normotensive and non-diabetic postmenopausal women were included as a control group (CG). None of the enrolled women was physically active. Electrocardiogram (ECG), PW tissue Doppler and transthoracic echocardiogram (TTE) were performed, baseline characteristics: demographic and clinical conditions are described in Table I.

The study was approved by the local bioethical committee and all patients gave their informed consent.

\section{Echocardiography}

Transthoracic echocardiogram was performed with patient in left lateral decubitus, after $10 \mathrm{~min}$ of resting, with the exam table elevated by $30^{\circ}$. The examination was carried out with $3.5 \mathrm{MHz}$ probe, with ECG trigger. We used echo-Doppler system equipped with a multifrequency transducer, GE Vivid T8, Health Care, via Galeno 126, Miland (Italy). We assessed: intraventricular septum thickness in diastole (IVSd), LV diastolic diameter (LVDD), LV posterior wall thickness during diastole (LVPWd), intraventricular septum thickness in systole (IVSs), LV systolic diameter (LVSD), LV posterior wall thickness during systole (LVPWs), ejection fraction (EF), and fractional shortening (FS). Peak velocities of early (E wave) and late (A wave) trans-mitral flow and deceleration time(DT) 
Table 1. Baseline characteristics (demographics, diseases and clinical conditions).

\begin{tabular}{lcccc}
\hline Demographic variables & All & Diabetic & Control group & P \\
\hline Age [\%] & & $56 \pm 13$ & $55 \pm 3$ & 0.3 \\
Postmenopausal women & 556 & 456 & 100 & \\
Body mass index $>30 \mathrm{~kg} / \mathrm{m}^{2}$ & 230 & $191(41.9 \%)$ & $39(39 \%)$ & 0.6 \\
HbA1c $>7.5 \%$ & 234 & $234(53.3 \%)$ & $0(0 \%)$ & \\
Abnormal electrocardiogram & 173 & $147(32.2 \%)$ & $26(26 \%)$ & 0.1 \\
\hline
\end{tabular}

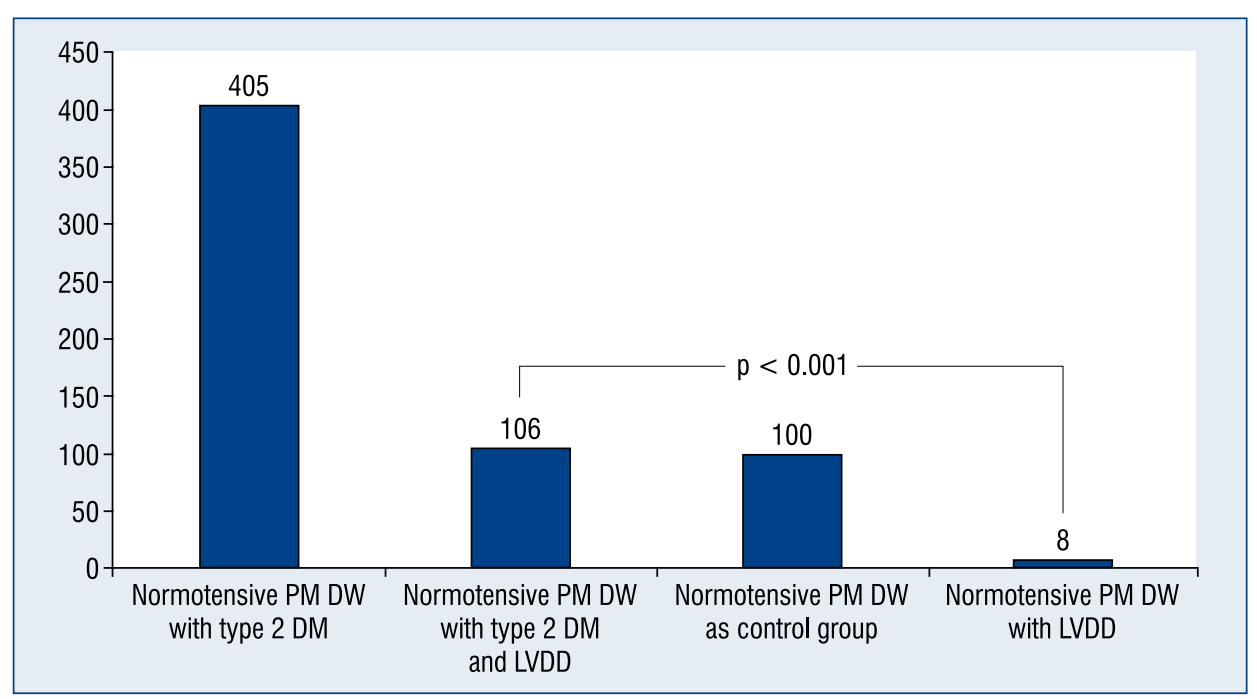

Figure 1. Left ventricular diastolic dysfunction (LVDD) in postmenopausal (PM) diabetic (D) normotensive women (W) according to diabetes status; DM — diabetes mellitus.

were determined, and E/A ratio was calculated. LV mass (LVM) was determined according to the formula by Devereux et al. [17] and indexed according to body surface area (BSA) to obtain LVM index (LVMI). Listed normal values of the above echocardiogram parameters are in accordance with the American Society of Echocardiography. LVDD was diagnosed by at least two expert cardiologists according to current guidelines [18, 19], by PW Doppler of mitral inflow and Doppler tissue imaging of the mitral annulus. LVDD was assessed as mild or grade I (impaired relaxation pattern), moderate or grade II (pseudonormal pattern), and grade III or severe (restrictive filling). Mild LVDD for mitral $\mathrm{E} / \mathrm{A}$ ratio $<0.8$, DT $>200 \mathrm{~ms}$, IVRT $\geq 100 \mathrm{~ms}$, $\mathrm{E}^{\prime}<8 \mathrm{~cm} / \mathrm{s}, \mathrm{E} / \mathrm{E}^{\prime}$ ratio $<8$ (septal and lateral). Moderate LVDD for mitral E/A ratio from 0.8 to 1.5 (pseudonormal), E/E' (average) ratio from 8 to 15 , and $\mathrm{E}^{\prime}<8 \mathrm{~cm} / \mathrm{s}$. Severe LVDD (grade III) for $\mathrm{E} / \mathrm{A}$ ratio $\geq 2, \mathrm{DT}<160 \mathrm{~ms}, \mathrm{IVRT} \leq 60 \mathrm{~ms}, \mathrm{E} / \mathrm{E}^{\prime}$ $>15$ [18]. All subjects with abnormal diastole, for all different degrees of severity, were assessed as having LVDD.

\section{Electrocardiogram}

It was assessed as abnormal according to observation of ST-T abnormalities at rest.

\section{Statistical analysis}

Statistical analysis was performed by IBM SPSS version 20.0 (Chicago, IL, USA). Results are described as mean with $95 \%$ confidence interval (CI 95\%). Student's t test was used for continuous variables and $\chi^{2}$ test for categorical variables. A p value $<0.05$ was considered significant.

\section{Results}

Left ventricular diastolic dysfunction was present in 103 (23.3\%) out of 456 diabetic women (DW), and 8 out of 100 women in CG (8\%), $\mathrm{p}<0.001$ (Fig. 1). There was no differ- 


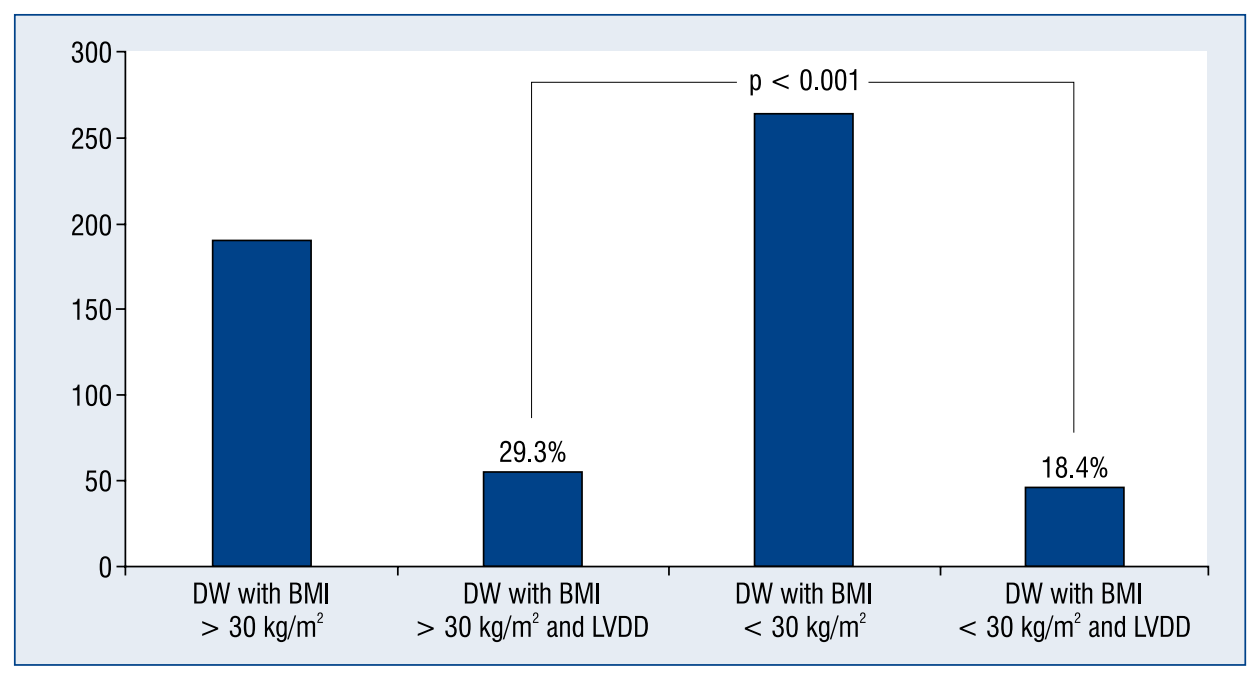

Figure 2. Left ventricular diastolic dysfunction (LVDD) in postmenopausal (PM) diabetic (D) normotensive women (W) according to different body mass index (BMI) and LVDD.

Table 2. Results according to left ventricular diastolic dysfunction distribution.

\begin{tabular}{lcccc}
\hline Clinical variables & Affected & Free & Control group & P \\
\hline Diabetic women & $105(23.3 \%)$ & $351(76.7 \%)$ & $8(8 \%)$ & $<0.001$ \\
Body mass index $>30 \mathrm{~kg} / \mathrm{m}^{2}$ & $56(12.3 \%)$ & $135(29.6 \%)$ & $39(39 \%)$ & $<0.005$ \\
Body mass index $<30 \mathrm{~kg} / \mathrm{m}^{2}$ & $49(10.7 \%)$ & $216(47.4 \%)$ & & $<0.01$ \\
HbA1c $>7.5 \%$ & $45(9.9 \%)$ & $198(43.4 \%)$ & $0(0 \%)$ & 0.1 \\
HbA1c $<7.5 \%$ & $15(3.3 \%)$ & $198(43.4 \%)$ & $100(100 \%)$ & $14(14 \%)$ \\
Abnormal electrocardiogram & $21(4.6 \%)$ & $126(27.7 \%)$ & $86(86 \%)$ & \\
Normal electrocardiogram & $84(18.4 \%)$ & $225(49.3 \%)$ & & \\
\hline
\end{tabular}

ence in mean age between the two groups: $56 \pm 13$ and $55 \pm 3$, respectively $(\mathrm{p}=0.3)$. There were $191(41.9 \%)$ DW with BMI $>30 \mathrm{~kg} /$ $/ \mathrm{m}^{2}$. Among those, there were $56(12.3 \%)$ with significant prevalence of LVDD, while there were $49(10.7 \%)$ with BMI $<30 \mathrm{~kg} / \mathrm{m}^{2}, \mathrm{p}<0.005$. Then, the number of DW with BMI $>30 \mathrm{~kg} / \mathrm{m}^{2}$ and normal diastolic function were 135 (29.6\%), DW with $\mathrm{BMI}<30 \mathrm{~kg} / \mathrm{m}^{2}$ and normal diastolic function were $216(47.4 \%)$. DW with HbA1c $>7.5 \%$ comprised a group of $243(53.3 \%)$ patients. Among those, there were $45(9.9 \%)$ with higher prevalence of LVDD, and 15 (3.3\%) with HbA1c < 7.5\%, p < 0.01 . A group of 198 (43.4\%) DW had poor glycemic control with normal diastolic function and equally, 198 (43.4.\%) DW had good glycemic control and normal diastolic function. Out of a group of $147(32.2 \%)$ DW with abnormal ECG, 21 had LVDD (4.6\%), $\mathrm{p}=0,1$, and 84 (18.8\%) had LVDD with normal ECG. Normal diastolic function was found in 126
(27.7\%) DW with abnormal ECG and in 225 (49.3\%) DW with normal ECG. All results are described in Table 2. $105 \mathrm{DW}$ affected by LVDD, 90 were on impaired relaxation pattern (85.7\%), 84 with normal ECG and 6 with abnormal. Eleven (10.4\%) DW were on pseudonormal pattern and $4(3.9 \%)$ on restrictive filling; all 15 DW had abnormal ECG. 8 women of CG affected by LVDD, all of them had abnormal ECG.

\section{Discussion}

Our study evaluated the prevalence of LVDD in asymptomatic and normotensive postmenopausal women, diabetic over 5 years. We found a higher prevalence of LVDD in our diabetic population compared with the CG (23.3\% for DW vs. $8 \%$ for CG). Other authors found LVDD as early subclinical myocardial damage in diabetic patients $[20,21]$. Furthermore (Fig. 2), we found a higher prevalence 
of LVDD in obese women with BMI $>30 \mathrm{~kg} /$ $/ \mathrm{m}^{2}$ (29.3\% for DW vs. $18.4 \%$ for CG) and with poor glycemic control, assessed by HbA1c $>7.5$ (35.4\% for DW vs. 28\% for CG) [22]. Bhuiyan et al. [23] observed a similar correlation between LVDD and uncontrolled DM. Our data are in accordance with Patil et al. [24], who found a strong correlation between LVDD and DM duration, $\mathrm{HbA1c}$ levels, obesity and microcirculation impairment. They underline that diabetic patients, although asymptomatic, within 10 years since first diagnosis, develop microcirculation impairment, which leads to cardiovascular events. Several mechanisms have been proposed to explain DM and LVDD correlation, such as increased cardiac lipid accumulation and impaired calcium homeostasis. LVDD but not systolic dysfunction was associated with increased cardiac triglyceride content in ob/ob mice [24]. Furthermore, these mice also exhibit impaired calcium reuptake that was associated with LV contractile dysfunction [25, 26]. Impaired contractility in cardiomyocytes isolated from sedentary $\mathrm{db} / \mathrm{db}$ mice was associated with increased diastolic sarcoplasmic reticulum (SR)- $\mathrm{Ca}^{2+}$ leak, reduced synchrony of $\mathrm{Ca}^{2+}$ release, lower peak systolic and diastolic $\mathrm{Ca}^{2+}$ and caffeine-induced $\mathrm{Ca}^{2+}$ release, consistent with a role for calcium in the LVDD seen in type $2 \mathrm{DM}$ [27]. This finding is in agreement with data concerning Indian population, genetically more prone to DM than others. It was assessed that LVDD is statistically associated with DM duration, poor glycemic control and mixed therapy consisting of insulin and oral hypoglycemic agents [28].

Another study, on 486 patients affected by $\mathrm{DM}$, found that the most important determinant of LVDD is DM duration over 4 years, regardless of hypertension and coronary artery disease affections [29]. Although DM duration seems to be a clear determinant for impaired LVDD, the prevalence of LVDD in newly-diagnosed (within 1 month) diabetic patients is high [30]. The prevalence of LVDD among diabetic population was $41 \%$, lower in women, regardless of confounding factors as hypertension, previous ischemia, and obesity. Poor glycemic control $(\mathrm{HbA} 1 \mathrm{c}>7.24 \pm 0.64)$ was statistically associated with LVDD. Age over 50 years at diagnosis was considered a major risk factor for LVDD development. However, in our opinion, high LVDD prevalence in newly-diagnosed diabetics seems due to a delay in diagnosis. Beyond diabetes, there are others determinants of LVDD: we found a strong correlation between LVDD and hypercholesterolemia in a population of postmenopausal women affected by a mild-moderate hypertension [31]. This finding can be easily explained by an increased aortic stiffness and high peripheral resistance that compromise $\mathrm{LV}$ diastolic function in postmenopausal women.

The main finding of this study consists in the evaluation of LVDD impact on asymptomatic postmenopausal women with normal blood pressure, assessing diabetes as a major determinant of LVDD. It is clear that hypertension is the most important factor for aortic stiffness, but it also plays an important role in determining LVDD [32]. Therefore, the diagnosis of LVDD in diabetic patients, without a confounding factor such as hypertension, requires LVDD monitoring, because it represents the first marker of "diabetic cardiomyopathy". Our study has found that poor glycemic control and obesity are additional factors for LVDD increase in diabetic population. The evidence of LVDD in diabetic normotensive post-menopausal women allows us to affirm that a complete echocardiographic examination in all diabetics is essential for an early diagnosis of "diabetic cardiomyopathy" [33]. LVDD diagnosis is often neglected in clinical practice in this subset of patients who are at high risk of developing adverse cardiac events. LVDD early diagnosis and treatment may be useful to reduce morbidity and improve the outcomes.

\section{Conclusions}

There is a high prevalence of LVDD in asymptomatic diabetic postmenopausal women; this finding is closely related with HbA1c levels and obesity status, not with abnormal ECG, which is a unique cardiologic test recommended by current guidelines in all diabetic subjects. We conclude that early detection of high level of HbA1c and obesity $\left(30 \mathrm{~kg} / \mathrm{m}^{2}\right)$ may identify women with major risk to develop LVDD. Furthermore, a simple ECG, when normal, is not enough to assess a normal LV diastolic function.

\section{Confict of interest: None declared}

\section{References}

1. Shi Y, Hu FB. The global implications of diabetes and cancer. Lancet, 2014; 383: 1947-1948.

2. Dodiyi-Manuel ST, Akpa MR, Odia OJ. Left ventricular dysfunction in normotensive type II diabetic patients in Port Harcourt, Nigeria. Vasc Health Risk Manag, 2013; 9: 529-533. 
3. Ciccone MM, Scicchitano P, Cameli M et al. Endothelial function in pre-diabetes, diabetes and diabetic cardiomyopathy: A review. J Diabetes Metab, 2014; 5: 4.

4. Dokainish H. Left ventricular diastolic function and dysfunction: Central role of echocardiography. Glob Cardiol Sci Pract, 2015; 2015: 3.

5. Nagueh SF, Appleton CP, Gillebert TC et al. Recommendations for the evaluation of left ventricular diastolic function by echocardiography. Eur J Echocardiogr, 2009; 10: 165-193.

6. Wan SH, Vogel MW, Chen HH. Pre-clinical diastolic dysfunction. J Am Coll Cardiol, 2014; 63: 407-416.

7. Owan TE, Hodge DO, Herges RM et al. Trends in prevalence and outcome of heart failure with preserved ejection fraction. $\mathrm{N} \mathrm{Engl}$ J Med, 2006; 355: 251-259.

8. Elesber AA, Redfield MM, Rihal CS et al. Coronary endothelial dysfunction and hyperlipidemia are independently associated with diastolic dysfunction in humans. Am Heart J, 2007; 153: 1081-1087.

9. Mishra TK, Rath PK, Mohanty NK, Mishra SK. Left ventricular systolic and diastolic dysfunction and their relationship with microvascular complications in normotensive, asymptomatic patients with type 2 diabetes mellitus. Indian Heart J, 2008; 60: 548-553.

10. Regitz-Zagrosek V, Oertelt-Prigione S, Seeland U, Hetzer R. Sex and gender differences in myocardial hypertrophy and heart failure. Circ J, 2010; 74: 1265-1273.

11. Zhao $Z$, Wang H, Jessup JA et al. Role of estrogen in diastolic dysfunction. Am J Physiol Heart Circ Physiol, 2014; 306: H628-H640.

12. Albu A, Fodor D, Bondor C, Poantă L. Arterial stiffness, carotid atherosclerosis and left ventricular diastolic dysfunction in postmenopausal women. Eur J Intern Med, 2013; 24: 250-254.

13. O'Rourke MF. The arterial pulse in health and disease. Am Heart J, 1971; 82: 687-702.

14. Palmiero P, Maiello M, Daly DD Jr et al. Aortic stiffness assessed by global pulse wave velocity in postmenopausal women: An ultrasonographic study. Echocardiography, 2012; 29: 1233-1238.

15. Coutinho T, Turner ST, Kullo IJ. Aortic pulse wave velocity is associated with measures of subclinical target organ damage. JACC Cardiovasc Imaging, 2011; 4: 754-761.

16. Maiello M, Zito A, Ciccone MM, Palmiero P. How aortic stiffness in postmenopausal women is related to common cardiovascular risk factors. Cardiol Res Pract, 2014; 2014: 216080.

17. Devereux RB, Alonso DR, Lutas EM et al. Echocardiographic assessment of left ventricular hypertrophy: comparison to necropsy findings. Am J Cardiol, 1986; 57: 450-458.

18. Nagueh SF, Appleton CP, Gillebert TC et al. Recommendations for the evaluation of left ventricular diastolic function by echocardiography. Eur J Echocardiogr, 2009; 10: 165-193.

19. Palmiero P, Zito A, Maiello M et al. Left ventricular diastolic function in hypertension: methodological considerations and clinical implications. J Clin Med Res, 2015; 7: 137-44. doi: 10.14740/jocmr2050w.
20. Raev DC. Which left ventricular function is impaired earlier in the evolution of diabetic cardiomyopathy? An echocardiographic study of young type I diabetic patients. Diabetes Care, 1994; 17: 633-639.

21. Stahrenberg R, Edelmann F, Mende M et al. Association of glucose metabolism with diastolic function along the diabetic continuum. Diabetologia, 2010; 53: 1331-1340.

22. Zabalgoitia M, Ismaeil MF, Anderson L, Maklady FA. Prevalence of diastolic dysfunction in normotensive, asymptomatic patients with well-controlled type 2 diabetes mellitus. Am J Cardiol, 2001; 87: 320-323.

23. Bhuiyan AS, Bari MA, Bari MS et al. Left ventricular diastolic dysfunction is more common in patients of uncontrolled diabetes mellitus. Mymensingh Med J, 2015; 24: 445-449.

24. Patil VC, Patil HV, Shah KB et al. Diastolic dysfunction in asymptomatic type 2 diabetes mellitus with normal systolic function. J Cardiovasc Dis Res, 2011; 2: 213-222.

25. Christoffersen C, Bollano E, Lindegaard ML et al. Cardiac lipid accumulation associated with diastolic dysfunction in obese mice. Endocrinology, 2003; 144: 3483-3490.

26. Van den Bergh A, Vanderper A, Vangheluwe P et al. Dyslipidaemia in type II diabetic mice does not aggravate contractile impairment but increases ventricular stiffness. Cardiovasc Res, 2008; 77: 371-379.

27. Dong $\mathrm{F}$, Zhang $\mathrm{X}$, Yang $\mathrm{X}$ et al. Impaired cardiac contractile function in ventricular myocytes from leptin-deficient ob/ob obese mice. J Endocrinol, 2006; 188: 25-36.

28. Stolen TO, Hoydal MA, Kemi OJ et al. Interval training normalizes cardiomyocyte function, diastolic $\mathrm{Ca} 2+$ control, and SR $\mathrm{Ca} 2+$ release synchronicity in a mouse model of diabetic cardiomyopathy. Circ Res, 2009; 105: 527-536.

29. Patil MB, Burji NP. Echocardiographic evaluation of diastolic dysfunction in asymptomatic type 2 diabetes mellitus. J Assoc Physicians India, 2012; 60: 23-26.

30. From AM, Scott CG, Chen HH. Changes in diastolic dysfunction in diabetes mellitus over time. Am J Cardiol, 2009; 103: 1463-1466.

31. Chaudhary AK, Aneja GK, Shukla S, Razi SM. Study on diastolic dysfunction in newly diagnosed type 2 diabetes mellitus and its correlation with glycosylated haemoglobin (HbA1C). J Clin Diagn Res, 2015; 9: OC20-OC22.

32. Palmiero P, Maiello M, Passantino A et al. Correlation between diastolic impairment and lipid metabolism in mild-to-moderate hypertensive postmenopausal women. Am J Hypertens, 2002; 15 (7 Part 1): 615-620.

33. Palmiero P, Maiello M, Daly DD et al. Relationship between global pulse wave velocity and diastolic dysfunction in postmenopausal women. Int J Clin Exp Med, 2014; 7: 5629-5635. eCollection 2014. 